\title{
The Ethics of Childhood Obesity Treatment - from the Childhood Obesity Task Force (COTF) of European Association for the Study of Obesity (EASO)
}

\author{
Jens Christian Holm ${ }^{a}$ Paulina Nowicka ${ }^{b}$ Nathalie J. Farpour-Lambert ${ }^{c}$ \\ Grace O'Malley $^{d, e}$ Maria Hassapidou ${ }^{f}$ Ram Weiss ${ }^{g}$ Jennifer L. Baker ${ }^{\text {h, }}{ }^{\mathrm{i}}$
}

\begin{abstract}
a The Children's Obesity Clinic, Department of Paediatrics, Copenhagen University Hospital Holbæk, Holbæk, Denmark, 'b Unit of Pediatrics, Department of Clinical Science, Intervention and Technology (CLINTEC), Karolinska Institute, Stockholm, Sweden, ' Department of Community Medicine and Primary Care, Geneva University Hospitals, Geneva, Switzerland, dPhysiotherapy Department, Temple Street Children's University Hospital, Dublin, Ireland, e Department of Epidemiology and Public Health, University College Cork, Cork, Ireland, ${ }^{f}$ Department of Nutrition and Dietetics, Alexander Technological Educational Institute of Thessaloniki, Thessaloniki, Greece, ${ }^{9}$ Department of Human Metabolism and Nutrition, Hebrew University, Jerusalem, Israel, hInstitute of Preventive Medicine, Bispebjerg and Frederiksberg Hospitals, The Capital Region, Copenhagen, Denmark, 'Novo Nordisk Foundation Center for Basic Metabolic Research, Faculty of Health and Medical Sciences, University of Copenhagen, Copenhagen, Denmark
\end{abstract}

Key Words

Child · Ethical issues · Observational study · Pediatric obesity · Randomized controlled trials

\section{Background}

With the American Medical Association's recent declaration that obesity is a serious disease [1], a critical consideration of the ethics of treating childhood obesity is especially timely. Although data suggest that the childhood obesity epidemic has stabilized in some countries [2], the levels remain too high; $18-49 \%$ of European children are overweight [3]. Consequently there is an immense and urgent need for the effective treatment of this complex disease. Unfortunately, the most recent Cochrane review concludes that, although familybased interventions can reduce excess weight in children and adolescents, long-term, effective and sustainable interventions for childhood obesity have yet to be identified [4]. 
Holm et al.: The Ethics of Childhood Obesity Treatment - from the Childhood Obesity Task Force (COTF) of European Association for the Study of Obesity (EASO)

Childhood obesity is a complex disease caused by a multitude of factors and it is associated with a wide range of co-morbidities. This complexity makes it challenging to design interventions and test the treatment approaches - particularly with regard to which outcome(s) should be used as the criteria for success. Most childhood obesity treatments occur in healthcare settings. As such, the randomized controlled trial (RCT), which is the 'gold standard' of the medical/clinical research tradition, has been used to evaluate whether a given obesity treatment protocol is safe and effective. It is questionable however, if the RCT is the optimal study design to address this complex issue.

Researchers investigating childhood obesity treatments face difficulties in deciding which outcomes best reflect the impact of treatment and, if a RCT is conducted, what type of treatment should it be randomized against. In light of the widespread childhood obesity epidemic, this paper will explore the ethical implications of these issues.

\section{Principles of Ethical Research in Children}

Pediatric obesity research is conducted in a vulnerable group of patients and families. Many children who are obese suffer from significant psychological problems including anxiety, depression, attention-deficit hyperactivity as well as emotional and eating disorders $[5,6]$. Added to this, obesity carries a social stigma that adversely affects children as well as their families. There has been - and still is - considerable debate as to who bears the responsibility for the development of excess weight in the child and who bears the responsibility for alleviating the condition at the individual and the societal levels [7, 8]. In recognition of these issues, there are discussions surrounding the ethics of community-based approaches of treating childhood obesity [9] and about mechanistic clinical studies aimed at understanding the pathophysiology of morbid obesity in children without a direct aim of reducing weight or the degree of obesity [10]. Less attention, however, has been given to the ethics of studying the individual treatment of the condition.

The vast majority of childhood obesity therapy research is conducted at hospitals and their associated clinics or at universities. In this framework, treatment protocols are examined and approved by ethical review committees; thus it can be assumed that the basic premises of these studies are ethically acceptable. Nonetheless, investigators are obliged to ensure that their research fulfils the four basic principles of ethical research: respect for autonomy, beneficence, non-maleficence, and justice [11]. In the context of childhood obesity research, this can be challenging. Children who are obese and their families are often desperate for treatment because existing programs can have long waiting lists or be out of reach due to cost, time, and transportation factors.

Given these circumstances, investigators must carefully consider if their protocol provides a situation in which they can scientifically test their hypotheses and balance this with creating a situation in which children and families freely make the choice to enter the study (autonomy), that the child receives a net benefit through participation in a timely manner (beneficence and non-maleficence), and that the exclusion criteria for participation are reasonable (justice). With these principles in mind, it becomes apparent that the development of treatment protocols must recognize the need for effective treatment along with consideration for the well-being of the child and his or her parent(s).

What Should Be the Primary Outcome of a Childhood Obesity Treatment Trial?

The majority of childhood obesity treatments use the reduction of weight, BMI $\left(\mathrm{kg} / \mathrm{m}^{2}\right)$, waist circumference, and/or body composition as the primary outcome. These studies usually evaluate the change over a period of a few months to a maximum of a couple of years. 
Although weight loss or stabilization represents a reasonable target, it may be overly simplistic since childhood obesity is a complex phenotype that is associated with multiple concurrent co-morbidities. Clinical studies suggest that almost $50 \%$ of children who are obese exhibit pre- or grade 1 or 2 hypertension [12,13], another $29 \%$ have dyslipidemia [14], $44 \%$ have more than $5 \%$ fat in their livers [15], and 74\% exhibit more than $5 \%$ fat in their muscles [16]. Additionally, children who are obese are at risk of serious cardiovascular [17, $18]$, endocrinological $[17,19]$, orthopedic $[20,21]$ and psychological $[5,6]$ complications that are present or not yet clinically apparent. Other complications progress silently and appear during late adolescence and young adulthood. Furthermore, future health risks loom, and a significantly increased risk of coronary heart disease [22], metabolic disease [23], and cancer $[24,25]$ is non-ignorable in adulthood.

Thus given the wide range of these co-morbidities and their potential for current and future harm to the child's health, addressing these problems individually without reducing the degree of obesity may be an acceptable and positive outcome of an obesity treatment. The reversal of adverse cardiovascular risk markers, such as increased blood pressure or dyslipidemia, is a clinical success, even without a reduction of obesity. Focusing the definition of 'success' on the isolated outcome of a statistically significant reduction in some aspect of body size ignores the patient's well-being, emotional status, and the plethora of other obesity-associated problems that are relevant for the health of the child.

\section{Hierarchy of Evidence}

In biomedical research, study designs can be ranked according to the strength of the evidence they can provide. Depending on the purpose - whether it is providing rapid treatment to a patient or to guiding a systematic review - there are different guidelines available [26]. One commonly used hierarchy ranks study designs based upon their internal validity [27]. Often depicted as a pyramid, RCTs are at the top, cohort studies are in the middle, and at the bottom are case reports [27]. In this scheme, controlled prospective trials in which subjects are not randomly allocated to the intervention but are compared with a control group fit somewhere just below RCTs.

Although hierarchies of evidence are convenient groupings of types of studies, they can be overly simplistic and cannot be applied to all situations. For example, it has been shown that well designed observational studies can achieve the same results as a RCT aimed at answering the same question, yet at a lower cost and with faster data collection and analysis [28]. Further, no matter how strong a study design is, the conclusions drawn from it arise from the scientific rigor applied to examining and ruling out other potential causes of the intervention effect. Similarly, even though these hierarchies are useful, they do not imply that the RCT is the best design for all research questions from scientific, practical or even ethical points of view.

\section{The Randomized Controlled Trial}

In biomedical research and for validation purposes before regulatory authorities, the RCT study design is viewed as the superior approach to establish whether an intervention is efficacious or not. Most commonly, one group is assigned to receive the intervention in question, whereas the other group receives a placebo or another treatment modality available in a common setting representing the current 'standard of care.' The RCT is well suited to studies of readily identifiable end points, such as pharmaceutical testing and surgical procedures. The design has high internal validity as well as the advantages of minimizing confounding, information and selection bias, and ruling out chance in the findings. Another 
Holm et al.: The Ethics of Childhood Obesity Treatment - from the Childhood Obesity Task Force (COTF) of European Association for the Study of Obesity (EASO)

advantage of this design is that it enhances the comparability of study results, especially if they are reported according to guidelines such as the CONSORT 2010 statement [29].

Inherent to the RCT design, however, is a limited generalizability of the results; a successful study in one setting does not guarantee that it can be replicated under normal conditions in another setting. Despite randomization, this study design does not eliminate confounding, but rather it allows for the measurement of the probability of confounding and thus an assessment of its effect on the outcomes of interest. Further, the RCT is a costly study design, which often limits the number of patients that can be included. This limitation has two consequences. First, a limited number of patients may be insufficient to actually test the effect of the intervention, and second, the inclusion of relatively small numbers of patients reduces the likelihood of observing side effects or complications of the treatment. Consequently, large-scale RCTs are difficult to finance. These limitations of the RCT design are especially relevant in the area of childhood obesity treatment where both the disease and its treatment are complex.

\section{RCTs and Childhood Obesity Treatment}

In the case of testing childhood obesity treatment programs, the traditional RCT study design is not optimal and may even fail. Allocation into simple treatment modalities does not reflect clinical practice or the true effects of treatment. Childhood obesity is a chronic disease without a well-defined clinical endpoint to evaluate; simply moving from a classification of obese to overweight, reducing BMI z-score, or losing some weight does not represent the end of a treatment requirement by the child. The RCT is not well suited for assessing this type of long-term outcome. In terms of generalizability, there are methodological problems regarding eligibility criteria since most children and youth selected for RCT-based childhood obesity treatment are highly motivated and thus are not comparable to the general population of children and youth who are obese. In addition, recruitment into such studies may be limited by cultural and societal beliefs as well as the level of education of parents [30, 31]. Moreover, these studies are typically performed in referral centers that treat children who are severely obese and may not be representative of the typical child who is 'mildly obese' or without a myriad of co-morbidities.

\section{Ethical Considerations of the RCT}

Aside from the limitations of the traditional RCT design in testing the efficacy of childhood obesity treatment protocols, it also presents an ethical dilemma of what care the control group should receive. In many childhood obesity interventions, the control group receives no treatment, is registered on a waiting list, or receives the standard care that is available at a given time (if indeed such a standard exists). From an ethical perspective, these approaches are problematic.

In the era of the childhood obesity epidemic, it is questionable if randomization to no treatment is acceptable. The Helsinki declaration of 2008 states that '... The benefits, risks burdens and effectiveness of a new intervention must be tested against those of the best current proven intervention except in the following circumstances: The use of placebo, or no treatment, is acceptable in studies where no current proven intervention exists ...' [32]. Narrowly interpreted, as there are not yet proven long-term, effective and sustainable interventions for the treatment of childhood obesity [33], a non-treatment control group could be considered as acceptable.

Viewed from a broader perspective, however, as there are interventions which produce the target outcome of weight loss or maintenance, even if it is not sustained for the long-term [4], the use of a non-treatment group is not acceptable. If one considers traditional diseases, most ethical committees would object to studies that randomize patients with diseases with 
known beneficial treatment options (such as cancer, diabetes, tuberculosis etc.) to no treatment. Yet, somehow, these standards are often not applied to childhood obesity. However, in recent years, given the extensive scope of the obesity epidemic and that children are becoming obese at progressively younger ages, many clinicians find this increasingly unethical and thus a violation of the Oath of Hippocrates.

As an alternative, subjects in the non-treatment arm of a childhood obesity treatment study should receive, at a minimum, standard care. In this context, since a universally accepted treatment has not been developed, providing it is a challenge. A justifiable approach to treating childhood obesity can consist of a familial approach, using behavior changing techniques, as well as simple advice concerning healthy nutrition and physical activity or inactivity [4, 34, 35]. The behavioral approach must be accompanied by medical intervention(s) for specific obesity-related co-morbidities, where appropriate. Nonetheless, the definition of standard care remains vague as these elements are often delivered in varying combinations and intensities and in different settings (personal, group, familial approach, or separation of the treatment modalities between patient and family etc.). Further compounding the problem is that in many settings even a minimal level of care for the obese child does not exist - in developed, let alone developing countries.

\section{Challenge of Evaluating Chronic Care}

In the evaluation of childhood obesity treatments, an implicit assumption is made that if an intervention is to be declared successful, it should result in a sustained treatment effect. In contrast, this requirement is not met by treatments given for nearly all other pediatric chronic diseases such as asthma, diabetes, or epilepsy. Patients with these serious chronic diseases tend to have relapses, which occur even with continuous medical care, but more rapidly without. Additionally, another challenge in the evaluation of chronic care models is that patients receive complex and individually tailored treatment designs (with varying visit intervals, focus of attention, use of different types of health professionals etc.) that are difficult to evaluate using current designs. Moreover, interventions designed for clinical trials may be much more intensive and costly than what is possible and attainable in normal clinical settings, particularly with regard to time, usage, and funding. The success of interventions such as these, due to their lack of 'real life' practicality, can result in frustration for practitioners who are unable to use these methods in their own setting. Unlike typical chronic medical conditions (such as hypertension or type 2 diabetes), the concept of childhood obesity may have cultural elements that should be incorporated and adapted into country-specific treatment and prevention programs - yet this significantly limits head-to-head evaluation of such interventions across Europe in empiric clinical trials.

When considering what standard care should consist of, it is worthwhile to remember that childhood obesity is a long-term condition with associated co-morbidities, many of which are not always readily identifiable. It is clear that children with chronic diseases need a relevant diagnosis followed by regular visits to monitor the progression, the emergence of complications, and the treatment responses alongside the continuous treatment given. Such standards are fundamental in good clinical practice. Chronic diseases demand chronic treatment models and need to be evaluated using study designs that can accommodate the complexity of both the disease and its treatment. These prerogatives are obvious and universally accepted in terms of chronic diseases in pediatrics, but not so in relation childhood obesity per se. Therefore, just as with the intervention arm of a trial, the care provided to these children should address these co-morbidities in addition to weight loss or maintenance. 


\section{Controlled Trials}

If we are to move beyond viewing the traditional RCT as the 'gold standard' in the evaluation of childhood obesity treatment programs, other designs must be explored. Such studies do not utilize a traditional RCT design, but rather employ a controlled trial design in which the comparison group is not randomly assigned and is often drawn from a source outside of the study. In this design, the most common sources of control subjects are: i) waiting lists, ii) usual care patients, and iii) a non-treatment group. Although appealing, many of these designs may not yield the comparison group that is expected by the researcher and each has ethical concerns, primarily that children who need treatment do not receive it.

Another alternative is 'The large simple trial' [36]. In this design, many institutions/ doctors join together in different settings so that thousands of patients can be included in a study at a lower cost and with better coverage of potential side effects (than compared with studies of hundreds of patients) [36]. In this case, a detailed recording of the therapeutic approaches, the duration and frequency of visits, health personals involved, and measurement methods have to be performed in order to compare treatments. Thus, to move forward in an ethically responsible manner, RCT trials testing childhood obesity treatments should be based upon comparisons between different therapeutic modalities.

\section{Observational Studies}

Multiple observational studies have been published regarding childhood obesity interventions. Despite the known limitations and biases of such studies, large observational sample evaluations can be used to analyze the effectiveness of pre-defined interventions for specific populations. Based on investigations in other medical fields, using this approach prudently can yield conclusions that are quite similar to those derived from large RCTs [28]

\section{Moving beyond the Traditional RCT}

Given the ethical considerations, the diversity in the obesity phenotypes that require complex and individualized treatment strategies as well as the long-term follow-up, the traditional RCT is not ideally suited for testing the efficacy of childhood obesity treatments. It is timely that the research community shift towards accepting study designs that demonstrate the plausibility of an intervention rather than just a probability statement derived from a RCT. 'Plausibility' refers to whether the intervention had an effect beyond what can be attributed to other external factors [37]. To make a strong plausibility statement, a control group still is required for comparison, but there is not a requirement that subjects are randomized to it. The commonly used sources (e.g. historical cohorts, waiting lists) can be used. A weaker statement can be made even if a control group is not used. Plausibility statements arise from the scientific rigor applied to examining and ruling out other potential causes for the treatment effect. Equally, the transparency of reports must increase, and the use of the CONSORT 2010 statement [29] would achieve this.

Childhood obesity is a complex condition with a multitude of causes and complications (some of which are not readily identifiable); thus relying only upon tests of probability as the sole criteria for establishing that an intervention was successful is not reasonable. It is time that results derived from adequately designed studies that use observational designs aimed at making causal statements are accepted. Given these constraints, this study design may be the only practical and ethical design option to answer clinical questions relevant to specific populations in Europe. Since obesity is a chronic condition with late emerging clinical sequelae, many years of follow-up are needed to truly appreciate the effectiveness of antiobesity interventions in children. This can rarely be achieved using RCTs and is unethical for the 'control' arm of such studies. 
Holm et al.: The Ethics of Childhood Obesity Treatment - from the Childhood Obesity Task Force (COTF) of European Association for the Study of Obesity (EASO)

\section{Conclusion}

In the evaluation of childhood obesity treatments it is time to move beyond viewing the RCT as the ideal study design in terms of evaluating treatment of clinically relevant childhood obesity. It is not well suited to test the complex and chronic care required to treat these children, and if the control group is randomized to no treatment at all, it is unethical. The time has come to provide standard care to all children who are obese and to evaluate treatment protocols accordingly by evaluating them in terms of their plausibility. Going forward, children and adolescents who are obese both need and deserve proper care.

\section{Disclosure Statement}

No conflicts of interest to declare.

\section{References}

1 American Medical Association: Is Obesity a Disease? (Resolution 115-A-12) Report of the Council on Science and Public Health, 2013, pp 14.

-2 Rokholm B, Baker JL, Sørensen TIA: The leveling off of the obesity epidemic since 1999 - a review of evidence and perspectives. Obes Rev 2010;11:835-846.

-3 Wijnhoven TM, van Raaij JM, Spinelli A, Rito AI, Hovengen R, Kunesova M, Starc G, Rutter H, Sjoberg A, Petrauskiene A, O’Dwyer U, Petrova S, Farrugia Sant'angelo V, Wauters M, Yngve A, Rubana IM, Breda J: WHO European Childhood Obesity Surveillance Initiative 2008: weight, height and body mass index in 6-9-year-old children. Pediatr Obes 2013;8:79-97.

4 Oude Luttikhuis H, Baur L, Jansen H, Shrewsbury VA, O'Malley C, Stolk RP, Summerbell CD: Interventions for treating obesity in children. Cochrane Database Syst Rev 2009:CD001872.

5 Puder JJ, Munsch S: Psychological correlates of childhood obesity. Int J Obes (Lond) 2010;34(suppl 2):S37-43.

-6 Russell-Mayhew S, McVey G, Bardick A, Ireland A: Mental health, wellness, and childhood overweight/obesity. J Obes 2012;2012:281801.

7 Holm S: Parental responsibility and obesity in children. Public Health Ethics 2008;1:21-29.

8 Wickins-Drazilova D, Williams G: Ethical and public policy aspects of childhood obesity: opinions of scientists working on an intervention study. Obes Rev 2010;11:620-626.

9 Perryman ML: Ethical family interventions for childhood obesity. Prev Chronic Dis 2011;8:A99.

$\checkmark 10$ Mann H: Ethics of research involving vulnerable populations. Lancet 2003;362:1857; author reply 18571858.

11 Gillon R: Medical ethics: four principles plus attention to scope. BMJ 1994;309:184-188.

12 Maggio AB, Aggoun Y, Marchand LM, Martin XE, Herrmann F, Beghetti M, Farpour-Lambert NJ: Associations among obesity, blood pressure, and left ventricular mass. J Pediatr 2008;152:489-493.

13 Holm JC, Gamborg M, Neland M, Ward L, Gammeltoft S, Heitmann BL, Sørensen TIA, Ibsen KK: Longitudinal changes in blood pressure during weight loss and regain of weight in obese boys and girls. J Hypertens 2012; 30:368-374.

14 Nielsen TR, Gamborg M, Fonvig CE, Kloppenborg J, Hvidt KN, Ibsen H, Holm JC: Changes in lipidemia during chronic care treatment of childhood obesity. Child Obes 2012;8:533-541.

15 Bille DS, Chabanova E, Gamborg M, Fonvig CE, Nielsen TRH, Thisted E, Thomsen HS, Holm JC: Liver fat content investigated by magnetic resonance spectroscopy in obese children and youths included in multidisciplinary treatment. Clin Obes 2012;2:41-49.

16 Fonvig CE, Bille DS, Chabanova E, Nielsen TR, Thomsen HS, Holm JC: Muscle fat content and abdominal adipose tissue distribution investigated by magnetic resonance spectroscopy and imaging in obese children and youths. Pediatr Rep 2012;4:e11.

17 Weiss R, Dziura J, Burgert TS, Tamborlane WV, Taksali SE, Yeckel CW, Allen K, Lopes M, Savoye M, Morrison J, Sherwin RS, Caprio S: Obesity and the metabolic syndrome in children and adolescents. N Engl J Med 2004; 350:2362-2374.

18 Aggoun Y, Farpour-Lambert NJ, Marchand LM, Golay E, Maggio AB, Beghetti M: Impaired endothelial and smooth muscle functions and arterial stiffness appear before puberty in obese children and are associated with elevated ambulatory blood pressure. Eur Heart J 2008;29:792-799.

19 O’Malley G, Santoro N, Northrup V, D’Adamo E, Shaw M, Eldrich S, Caprio S: High normal fasting glucose level in obese youth: a marker for insulin resistance and beta cell dysregulation. Diabetologia 2010;53:1199-1209. 
Holm et al.: The Ethics of Childhood Obesity Treatment - from the Childhood Obesity Task Force (COTF) of European Association for the Study of Obesity (EASO)

20 Jannini SN, Doria-Filho U, Damiani D, Silva CA: Musculoskeletal pain in obese adolescents. J Pediatr (Rio J) 2011;87:329-335.

21 O'Malley G, Hussey J, Roche E: A pilot study to profile the lower limb musculoskeletal health in children with obesity. Pediatr Phys Ther 2012;24:292-298.

-22 Baker JL, Olsen LW, Sørensen TIA: Childhood body-mass index and the risk of coronary heart disease in adulthood. N Engl J Med 2007;357:2329-2337.

23 Juonala M, Magnussen CG, Berenson GS, Venn A, Burns TL, Sabin MA, Srinivasan SR, Daniels SR, Davis PH, Chen W, Sun C, Cheung M, Viikari JS, Dwyer T, Raitakari OT: Childhood adiposity, adult adiposity, and cardiovascular risk factors. N Engl J Med 2011;365:1876-1885.

24 Kitahara CM, Gamborg M, Berrington de Gonzalez A, Sørensen TIA, Baker JL: Childhood height and body mass index were associated with risk of adult thyroid cancer in a large cohort study. Cancer Res 2014;74:235-242.

25 Berentzen TL, Gamborg M, Holst C, Sørensen TIA, Baker JL: Body mass index in childhood and adult risk of primary liver cancer. J Hepatol 2014;60:325-330.

26 Howick J, Chalmers I, Glasziou P, Greenhalg T, Heneghan C, Liberati A, Moschetti I, Philips B, Thornton H: Explanation of the 2011 Oxford Centre for Evidence-Based Medicine (OCEBM) Levels of Evidence (Background Document). Oxford, Oxford Centre for Evidence-Based Medicine, 2011.

27 Ho PM, Peterson PN, Masoudi FA: Evaluating the evidence: is there a rigid hierarchy? Circulation 2008;118: $1675-1684$.

28 Concato J, Shah N, Horwitz RI: Randomized, controlled trials, observational studies, and the hierarchy of research designs. N Engl J Med 2000;342:1887-1892.

29 Schulz KF, Altman DG, Moher D; CONSORT Group: CONSORT 2010 statement: updated guidelines for reporting parallel group randomised trials. BMJ 2010;340:c332.

-30 Skelton JA, Irby MB, Beech BM, Rhodes SD: Attrition and family participation in obesity treatment programs: clinicians' perceptions. Acad Pediatr 2012;12:420-428.

-31 Leech RM, McNaughton SA, Timperio A: Clustering of children's obesity-related behaviours: associations with sociodemographic indicators. Eur J Clin Nutr 2014;68:623-628.

32 World Medical Association: WMA Declaration of Helsinki - Ethical Principles for Medical Research Involving Human Subjects. Seoul, 59th WMA General Assembly, October 2008.

-33 Waters E, de Silva-Sanigorski A, Hall BJ, Brown T, Campbell KJ, Gao Y, Armstrong R, Prosser L, Summerbell CD: Interventions for preventing obesity in children. Cochrane Database Syst Rev 2011;12:CD001871.

-34 Barlow SE: Expert committee recommendations regarding the prevention, assessment, and treatment of child and adolescent overweight and obesity: summary report. Pediatrics 2007;120(suppl 4):S164-192.

35 Nowicka P, Flodmark CE: Family therapy as a model for treating childhood obesity: useful tools for clinicians. Clin Child Psychol Psychiatry 2011;16:129-145.

36 Sackett DL: Clinical Epidemiology: A Basic Science for Clinical Medicine, 3rd ed. Boston, Little, Brown, 1991.

-37 Habicht JP, Victora CG, Vaughn JP: Evaluation designs for adequacy, plausibility and probability of public health programme performance and impact. Int J Epidemiol 1999;28:10-18. 